\title{
Biofeedback Benefits Only Patients With Outlet Dysfunction, Not Patients With Isolated Slow Transit Constipation
}

\author{
GIUSEPPE CHIARIONI,* LARA SALANDINI,* and WILLIAM E. WHITEHEAD ${ }^{\dagger}$ \\ *Divisione di Riabilitazione Gastroenterologica, Universitá di Verona, Azienda Ospedaliera di Verona, Centro Ospedaliero Clinicizzato, \\ Valeggio sul Mincio, Verona, Italy; and ${ }^{*}$ UNC Center for Functional Gastrointestinal and Motility Disorders, and Division of Gastroenterology \\ and Hepatology, University of North Carolina at Chapel Hill, Chapel Hill, North Carolina
}

Background \& Aims: Biofeedback is reported to be as effective for slow transit constipation as for pelvic floor dyssynergia and no more effective than education. We aimed to test the hypothesis that biofeedback benefits only patients with pelvic floor dyssynergia, describe the physiologic mechanism of treatment, and identify predictors of success. Methods: Fifty-two patients (49 women; average age, 35 years), all with delayed whole gut transit, included 34 with pelvic floor dyssynergia, 12 with slow transit only, and 6 who met only 1 of 2 criteria for pelvic floor dyssynergia. All received 5 weekly biofeedback sessions directed at increasing rectal pressure and relaxing pelvic floor muscles during straining plus practice defecating a balloon. Patients were retested by questionnaire; symptom diary; balloon defecation; transit study at 1, 6, 12, and 24 months; and anorectal manometry at 1 and 6 months. Results: At 6 months, greater improvements were seen in pelvic floor dyssynergia compared with slow transit only; $71 \%$ versus $8 \%$ reported satisfaction $(P=.001)$, and $76 \%$ versus $8 \%$ reported $\geq 3$ bowel movements per week $(P<.001)$. Improvements were maintained at $\mathbf{2 4}$ months of followup. Biofeedback eliminated dyssynergia in $91 \%$ and enabled $85 \%$ to defecate the balloon. Satisfaction was correlated with improved ability to defecate the balloon $(\rho=.73 ; P<.001)$, reductions in dyssynergia $(\rho=.69$; $P<.001)$, and increased rectal pressure during straining $(\rho=.36 ; P<.01)$. Success was predicted by pelvic floor dyssynergia, milder constipation, and less frequent abdominal pain at baseline. Conclusions: Biofeedback is an effective treatment for pelvic floor dyssynergia but not slow transit constipation.

C onstipation is a symptom that affects $12 \%-19 \%$ of adults in the United States. ${ }^{1}$ Several pathophysiologic mechanisms are recognized that may cause constipation, including megacolon or megarectum (dilated, atonic bowel) and drug side effects. However, the most commonly recognized types of constipation are slow transit constipation and outlet dysfunction. ${ }^{2}$

Slow transit constipation is defined as delayed transit throughout the colon and is believed to result from reduced numbers of high-amplitude propagating contractions ${ }^{3,4}$ associated with decreased numbers of interstitial cells of Cajal.5,6 Diagnosis is based on transit studies showing abnormally prolonged transit of radiopaque markers or radioisotopes through the colon..$^{7,8}$

Outlet dysfunction-type constipation refers to difficulty evacuating the rectum. Preston and Lennard-Jones ${ }^{9}$ were the first to describe this type of constipation; they showed that a subgroup of patients with constipation failed to relax the pelvic floor muscles during defecation and instead paradoxically contracted these muscles. Subsequent investigators supported their observation, ${ }^{10}$ and biofeedback techniques were developed to teach patients to evacuate the rectum by relaxing the pelvic floor muscles. ${ }^{11,12}$ However, Duthie and Bartolo ${ }^{13}$ found that up to $80 \%$ of constipated patients who exhibit paradoxical contraction of the pelvic floor muscles in the gastroenterology clinic may show normal pelvic floor relaxation during defecation at home, and others have noted that a significant proportion of asymptomatic people show paradoxical contraction of the pelvic floor when tested in the clinic. Rao et $\mathrm{al}^{14}$ showed that inadequate intrarectal pressure during attempts at defecation may also explain failure to evacuate the rectum. These observations have led to controversy over whether pelvic floor dyssynergia is a distinct mechanism for constipation.

Biofeedback training to teach relaxation of the pelvic floor was reported to be effective in a number of trials, ${ }^{12,15,16}$ with the median response rate approximately $67 \% .{ }^{12}$ However, there have been no randomized controlled trials to confirm efficacy in adults. The St Mark's group $^{17-19}$ reported that biofeedback was equally effective in patients with slow transit constipation and those with outlet dysfunction characterized by paradoxical con-

\footnotetext{
Abbreviations used in this paper: ANCOVA, analysis of covariance MIX, mixed; PFD, pelvic floor dyssynergia with slow transit; STO, slow transit only.

(C) 2005 by the American Gastroenterological Association $0016-5085 / 05 / \$ 30.00$ doi:10.1053/j.gastro.2005.05.015
} 
traction of the pelvic floor and that biofeedback training resulted in acceleration of whole gut transit time in patients with slow transit constipation. This claim of equal efficacy in slow transit constipation and outlet dysfunction constipation was supported by an independent research group. ${ }^{20}$ The St Mark's group also reported that biofeedback was no more effective than education and training performed with verbal feedback but without instruments to assist patients in learning to relax the pelvic floor. ${ }^{17}$ These 2 observations, that biofeedback to teach relaxation of the pelvic floor is as effective in patients who do not demonstrate pelvic floor dysfunction as a cause of their constipation as it is in patients with pelvic floor dyssynergia and that visual feedback is no more effective than education, challenge claims that biofeedback training makes a specific contribution to the treatment of constipation that is distinct from education or psychotherapy.

The primary aim of this study was to determine whether biofeedback to teach relaxation of the pelvic floor muscles benefits only patients with outlet dysfunction-type constipation versus whether, as claimed by the St Mark's group, it improves constipation equally well in patients with slow transit constipation who do not have outlet dysfunction. Secondary aims were to determine whether physiologic parameters identify the mechanism of biofeedback training effects (ie, whether clinical benefits of biofeedback are related to changes in pelvic floor physiology or are nonspecific) and to determine whether physiologic parameters or clinical symptoms recorded at baseline identify the patients who are most likely to respond to biofeedback training.

\section{Materials and Methods}

\section{Patients}

Seventy-five consecutive patients referred to the gastroenterology clinic of the Division of Gastrointestinal Rehabilitation of the University of Verona at Valeggio s/M Hospital, Azienda Ospedaliera di Verona, for refractory, long-standing (at least 12 months) constipation between June 1997 and January 2001 were screened for the study. All patients were evaluated by history, complete medical examination, biochemical studies to exclude secondary causes of constipation such as hypothyroidism and drug side effects, and a barium enema study to identify those with megacolon or megarectum. All patients were then enrolled into a 1-month run-in phase to confirm that they met inclusion criteria (see following text). The study was conducted in compliance with the Declaration of Helsinki.

\section{Run-in Period}

For 30 days, all patients were treated with conservative medical management consisting of $30 \mathrm{~g} /$ day of fiber supple- mentation. They were allowed to take laxatives or enemas during this run-in period. All patients were asked to keep a symptom diary in which they recorded (1) laxative and/or enema use, (2) number of bowel movements, (3) straining at stool, (4) experience of severe pain, and (5) experience of severe bloating. Pain and bloating were defined as severe if they were intense enough to interfere with usual daily activities. At the end of the 1-month run-in period, patients' diaries were reviewed. Five patients were excluded ( 2 for not keeping the diary and 3 for almost-daily consumption of laxatives). The remaining 70 patients underwent a Sitzmark test ${ }^{21}$ of whole gut transit while abstaining from laxatives and enemas (see following text).

\section{Inclusion/Exclusion Criteria}

To be included, patients had to (1) report $<3$ bowel movements per week by history, (2) fail a test of a high-fiber diet (at least $30 \mathrm{~g} /$ day for 30 days during the run-in period), and (3) have an abnormally delayed whole gut transit study defined as $\geq 5$ of 20 Sitzmarks remaining in the colon 5 days after ingestion. Patients were enrolled regardless of whether they reported anorectal symptoms suggestive of outlet dysfunction. Patients were excluded if they had (1) previous abdominal surgery except for appendectomy; (2) megacolon, megarectum, or hypothyroidism; or (3) resolution of symptoms on conservative medical management (fiber supplementation).

Fifty-two patients who satisfied inclusion and exclusion criteria were invited to participate in a behavioral therapy trial, and all agreed and signed an informed consent statement. These 52 patients with slow transit constipation included 49 women and 3 men with an average age of $34.9 \pm 10.2$ years (mean $\pm \mathrm{SD}$; range, 23-63 years).

\section{Study Design}

Figure 1 shows the study design. Patients who satisfied inclusion/exclusion criteria at the end of the run-in phase were evaluated by anorectal manometry and balloon defecation tests and were classified as either slow transit only (STO), pelvic floor dyssynergia with slow transit (PFD), or mixed (MIX). The STO group included patients who retained $\geq 5$ Sitzmarks 5 days after ingesting 20 of them and who satisfied neither of the 2 criteria for PFD. The PFD group included patients with slow transit constipation ( $\geq 5$ of 20 Sitzmarks remaining 5 days after ingestion) who met both of 2 criteria: paradoxical contraction or failure to relax the pelvic floor muscles during attempts to defecate and inability to evacuate a $50-\mathrm{mL}$ waterfilled balloon within 5 minutes. The MIX group included patients with slow transit constipation who satisfied one but not both of the criteria for PFD. All patients (regardless of diagnostic group assignment) received 5 sessions of biofeedback training to teach relaxation of the pelvic floor during defecation and were then followed up by repeat manometry, balloon defecation, Sitzmark study of transit, 1-month symptom diary, and ratings of satisfaction with treatment outcome at clinic visits 1 month and again 6 months after the conclu- 


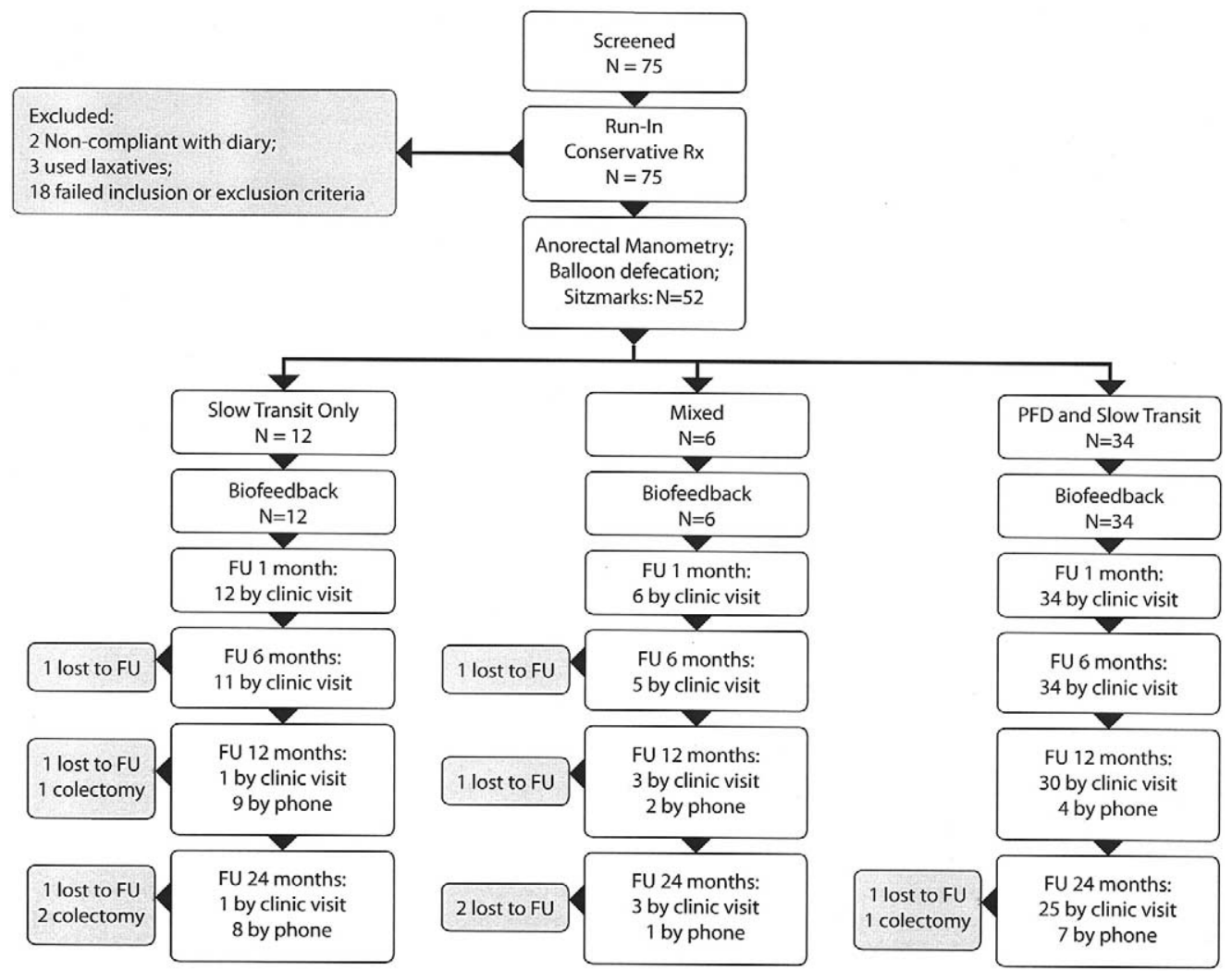

Figure 1. Study design.

sion of biofeedback training. With few exceptions (noted in Figure 1), the PFD group returned to the clinic to provide satisfaction ratings and kept a 1-month symptom diary at 12 months and 24 months of follow-up but were not asked to repeat the anorectal manometry test at 12 months and 24 months. When any patient declined to return to the clinic for follow-up or to keep 1-month symptom diaries at 12 months or 24 months, follow-up data on satisfaction with treatment were collected by telephone.

\section{Sitzmark Study of Whole Gut Transit Time}

The Hinton technique ${ }^{21}$ was used to measure whole gut transit time. Patients ingested a gelatin capsule containing 20 radiopaque soft rubber markers (Sitzmarks; Konsyl Pharmaceuticals, Fort Worth, TX), and a single abdominal radiograph was taken on the fifth day. Patients were asked to consume $30 \mathrm{~g} /$ day of fiber supplement during the week preceding and the week of the Sitzmark study. The results of the study were said to be abnormal if $\geq 5$ of 20 markers remained in the colon at day 5. Rectal stasis was defined by the patient retaining more than 10 markers at day 5 , with more than $80 \%$ of them located in the rectosigmoid area.

\section{Balloon Defecation Test}

A flexible plastic catheter (10-cm long; OD, $3 \mathrm{~mm}$ ) with a latex balloon made from a condom (SSL Healthcare Italia, Bologna, Italy) on its tip was lubricated and inserted into the rectum. This balloon was $5 \mathrm{~cm}$ long by $3 \mathrm{~cm}$ wide when deflated and $5 \mathrm{~cm}$ long by $3.5 \mathrm{~cm}$ in diameter (approximately cylindrical in shape) when inflated with $50 \mathrm{~mL}$ of water. After insertion, the balloon was filled with $50 \mathrm{~mL}$ of water at room temperature, and the patient was instructed to sit on a commode chair in a room by themselves and to pass the balloon. Up to 5 minutes was allowed for defecation, and any patient who was unable to pass the balloon was judged to have an abnormal balloon defecation test result.

\section{Anorectal Manometry}

The manometry catheter (model R6B; Mui Scientific, Missisauga, Ontario, Canada) had a latex balloon on its tip that could be distended with air via a handheld syringe, and it had 4 perfusion ports spaced $1 \mathrm{~cm}$ apart beginning $2 \mathrm{~cm}$ below the balloon to measure pressures. The inner diameter of each of the 4 perfusion catheters was $0.8 \mathrm{~mm}$, and they were perfused with degassed water at a rate of $0.5 \mathrm{~mL} / \mathrm{min}$ by a low-compliance pump (Arndorfer Medical Specialties, Greendale, WI). The outer diameter of the catheter was $4.5 \mathrm{~mm}$. Pressures were recorded and displayed using a model GR 800 polygraph (Aspen Medical, Dingwall, Scotland). Pressure recordings were analyzed manually.

With the patient in the left lateral position, the manometry cathether was lubricated and inserted into the rectum. It was then pulled back in $0.5-\mathrm{cm}$ steps, and pressures were recorded at each position while the patient was instructed to relax. The peak pressure (averaged across all 4 perfusion ports) defined anal canal resting pressure. The second perfusion port was then 
positioned in the high-pressure zone of the anal canal, and the rectal balloon was distended with varying volumes of air (50, $40,30,20,15,10$, and $5 \mathrm{~mL}$ ) to determine the smallest volume of distention that elicited a rectoanal inhibitory reflex (defined as the reflex decrease in anal canal pressure that is elicited by rectal distention). Next, the rectal balloon was inflated in $20-\mathrm{mL}$ steps up to $500 \mathrm{~mL}$ to assess the threshold for the sensation of urge to defecate and the maximum tolerable volume. Compliance was defined as the rectal balloon pressure at $100-\mathrm{mL}$ distention. The peak pressure in the rectum during straining to defecate was recorded and defined as straining pressure. ${ }^{22}$ Finally, the patient was asked to strain to pass the rectal balloon and was considered dyssynergic if anal canal pressure during straining demonstrated either a paradoxical contraction or a failure to decrease (relax). A phosphate enema was administered approximately 2 hours before the anorectal manometry and balloon defecation tests.

\section{Definition of Pelvic Floor Dyssynergia}

Patients were categorized as PFD if they satisfied both of 2 criteria: (1) dyssynergia on manometry and (2) inability to defecate a $50-\mathrm{mL}$ water-filled balloon in 5 minutes. Both criteria were met by 34 patients who were classified as PFD. Twelve patients fulfilled neither of these criteria and were classified as STO. Six patients were discordant; 2 demonstrated dyssynergia on manometry but were able to defecate the balloon, and 4 could not defecate the balloon but had normal manometry. These 6 discordant patients were classified as MIX and were analyzed separately.

\section{Biofeedback Training}

All 52 patients meeting inclusion criteria were told that their constipation could be related to a defecation disorder and that improving the defecation process might help them. All of them underwent 5 weekly biofeedback training sessions that lasted 30-45 minutes. All biofeedback training was done by one of the investigators (G.C.), who was not aware of the results of the manometry and balloon expulsion tests.

Biofeedback training consisted of 3 phases. In the first phase, patients were taught to strain more effectively and to coordinate expulsion efforts with their breathing. This was usually accomplished in the first session. In the second phase of training, a portable, battery-operated electromyographic instrument (Myotron-120; Enting Instruments \& Systems, Dorst, The Netherlands) was used to teach the patient to relax the sphincter during straining. The anal plug had a bulb on its end to hold it in position, and it had 3 longitudinally oriented metal plates on its surface to record electromyography from the anal canal. The electromyographic signal was averaged and then displayed on a monitor in microvolts. Patients watched the display while contracting and relaxing the anal sphincter. They were told that higher numbers were related to closure of the sphincter and smaller numbers were associated with opening the sphincter and that the lower the numbers were during straining, the more effective defecation would become.
In the third phase of training, patients practiced defecating an air-filled balloon attached to a catheter. With the patient laying on his or her left side, the catheter was placed into the rectum and inflated with $50 \mathrm{~mL}$ of air. The inflated balloon was gently pulled out of the rectum by the investigator while the patient attempted to avoid contracting the sphincter. On successive trials, the amount of assistance from the therapist (amount of traction on the catheter) was gradually decreased as the patient learned to strain effectively to expel the inflated ballon.

All 3 phases of training had to be accomplished in 5 sessions, with at least one session of balloon feedback. At the end of therapy, all patients were told that their pushing effort had improved and that they had to be followed up to monitor the effects of this training on their symptoms. All patients were asked to continue on a high-fiber diet and to avoid using laxatives as much as possible.

\section{Follow-up}

Anorectal manometry, the balloon expulsion test, and the Sitzmark transit study were repeated 1 and 6 months after completion of therapy. All patients were also asked to report to the clinic 12 months and again 24 months after the end of therapy, and the balloon expulsion test and Sitzmark transit study were repeated. (However, for the most part, only responders returned for 12-month and 24-month follow-up.) One month before the clinic appointment, patients were reminded by telephone to keep a 30-day symptom diary in which they reported the same parameters as during the run-in period.

\section{Clinical Outcome Measures}

The primary outcome measure was the patients' rating of their overall improvement. All patients were asked, either at their follow-up clinic visit or by telephone if they did not return to the clinic, to rate their overall improvement by responding to the following question: "How would you grade your symptom improvement: none (1), mild (2), fair (3), or major (4)." In addition, patients were asked to keep a 30-day symptom diary before each follow-up visit in which they recorded each day the number of bowel movements, occurrence of excessive straining with bowel movements, use of laxatives, occurrence of pain, and occurrence of bloating. The average number of these events per week was used in data analysis.

\section{Physiologic Outcome Measures}

Whole gut transit time and ability to defecate a $50-\mathrm{mL}$ water-filled balloon were evaluated at baseline and at each follow-up interval. Anorectal manometry parameters were recorded at baseline, 1-month follow-up, and 6-month follow-up and included the following: resting pressure in the anal canal, rectoanal inhibitory reflex, urge threshold, maximum tolerable volume, compliance, rectal pressure during straining, and presence of dyssynergia. Physiologic parameters (including judgments about whether dyssynergia was present) were made 
by one investigator who was blind to the results of the Sitzmark study and the balloon defecation test.

\section{Statistical Analysis}

In all analyses of treatment efficacy, all 52 patients who met inclusion criteria and entered the trial were retained in the analyses, and all 3 groups were included. However, follow-up tests (paired comparisons) compared only the STO group with the PFD group because this provided the most unambiguous test of the hypotheses. As shown in Figure 1, there were few missing data for the primary outcome measure (improvement ratings); these were dealt with by carrying forward the last available observation.

The primary outcome variable was an ordinal scale rating ranging from 1 to 4 . Because this variable was not continuous or normally distributed, the primary test of the hypothesis was a Kruskall-Wallis test comparing STO, MIX, and PFD at each follow-up interval. Follow-up paired comparisons between the STO and PFD groups used the Mann-Whitney $U$ test. Average ratings were also computed for graphical representation.

All continuous variables were analyzed by repeated-measures analysis of covariance (ANCOVA) using the SPSS general linear models program, ${ }^{23}$ followed by $t$ tests comparing the STO group with the PFD group at each time point. Age was entered as a covariate in these analyses because age differences between the PFD and STO groups approached significance ( $P$ $=.053$ ). Dichotomous variables (balloon defecation and presence of dyssynergia) were analyzed by separate $\chi^{2}$ tests comparing STO with PFD at baseline and at each follow-up interval. An $\alpha$ level of .05 was used for all statistical tests.

One of our secondary aims was to determine whether biofeedback treatment effects were related to changes in physiology (the goal of biofeedback training) or were nonspecific. To assess this, we pooled all 52 patients and calculated changes in physiologic parameters from baseline to the 1-month and 6-month follow-up assessments and then computed the Spearman nonparametric correlations between these change scores and patient ratings of satisfaction with treatment at 1-month and 6-month follow-up. We also used ordinal regression analysis to determine which of the variables identified by significant univariate correlations made significant independent contributions to the prediction of successful biofeedback training after adjusting for the intercorrelations among these variables.

Another secondary aim was to determine whether there were baseline physiologic or clinical characteristics that could identify which patients were most likely to receive a clinical benefit from biofeedback training. For these analyses, all 52 patients were pooled and the nonparametric Spearman correlation between the treatment satisfaction rating at 6 months and all physiologic and clinical characteristics recorded at baseline was computed. We also used ordinal regression analysis to determine which of the baseline variables identified by significant univariate correlations made significant independent contributions to the prediction of successful biofeedback training after adjusting for the intercorrelations among these variables.

\section{Results \\ Demographics}

The 12 patients in the STO group included 2 men, the 34 patients in the PFD group included one man, and the 6 patients in the MIX group included no men. The average (mean $\pm \mathrm{SD}$ ) age of patients in the STO group was $39.6 \pm 11.8$ years, compared with 36.7 \pm 10.3 years for patients in the MIX group and $32.9 \pm$ 9.3 years for patients in the PFD group. Differences in age between the STO and PFD groups approached significance $(t[44]=1.99 ; P=.053)$. Although no exclusion criteria on the basis of age, race, or other personal factors were used, all patients were Italians of European descent.

\section{Clinical Characteristics}

All patients reported frequent use of laxatives or enemas, and all reported that they had needed to gradually increase their use of laxatives to achieve defecation. Their average duration of constipation was 14 years (range, 2-40 years). Digital facilitation of defecation was volunteered by 8 patients, of whom 7 were unable to defecate a 50-mL water-filled balloon.

\section{Patients Lost to Follow-up}

As shown in Figure 1, all patients attended the 1-month follow-up, but 2 patients (one in the STO group and one in the MIX group) did not return for the 6-month follow-up. Both were nonresponders; when contacted by telephone at 6 months, one was consulting a local surgeon for colectomy (STO group) and the other had been in an automobile accident and sustained a pelvic fracture (MIX group). She was lost to subsequent follow-up. The same 2 patients plus one additional STO patient failed to return for the 12-month follow-up. At 24 months, one patient in the STO group could not be contacted and 2 patients had undergone a colectomy. At 24 months, 2 patients in the MIX group could not be contacted. In the PFD group, one patient was lost to follow-up and one patient had undergone a colectomy by the 24-month follow-up.

\section{Satisfaction With Treatment}

Figure 2 shows average satisfaction ratings at each follow-up interval. Patients in the PFD group rated their satisfaction with treatment significantly $(P \leq .001)$ higher than patients in the STO group at each follow-up interval. Seventy-one percent of patients in the PFD group rated their satisfaction with treatment as "fair" or "major" at all follow-up intervals compared with $8 \%$ of 




Figure 2. Satisfaction ratings (mean $\pm \mathrm{SE}$ ). Open bars, STO group; gray bars, MIX group; black bars, PFD group. 1, none; 2, mild; 3, fair; 4 , major satisfaction with treatment. All comparisons are significant at $P \leq .001$.

patients in the STO group and $50 \%$ of patients in the MIX group $(P=.001)$.

\section{Stool Frequency}

Figure 3 shows the average frequency of bowel movements at baseline and each follow-up interval. Repeated-measures ANCOVA showed a significant interaction between groups and time periods (GreenhouseGeisser corrected F[3.89, 93.32] $=7.23 ; P<.001)$. Paired comparisons showed that there was no difference between the groups at baseline; however, at each subsequent follow-up interval, patients in the PFD group had significantly $(P<.001)$ more bowel movements than patients in the STO group. At 6-month follow-up, $76 \%$ of patients in the PFD group were having $\geq 3$ bowel movements per week compared with $8 \%$ of patients in the STO group $(P<.001)$; this difference persisted at 12-month follow-up (82\% vs $8 \%)$ and 24-month follow-up (79\% vs $8 \%)(P<.001$ for each comparison). At baseline, $12 \%$ of patients in the PFD group and none of the patients in the STO group reported having $\geq 3$ bowel movements per week.

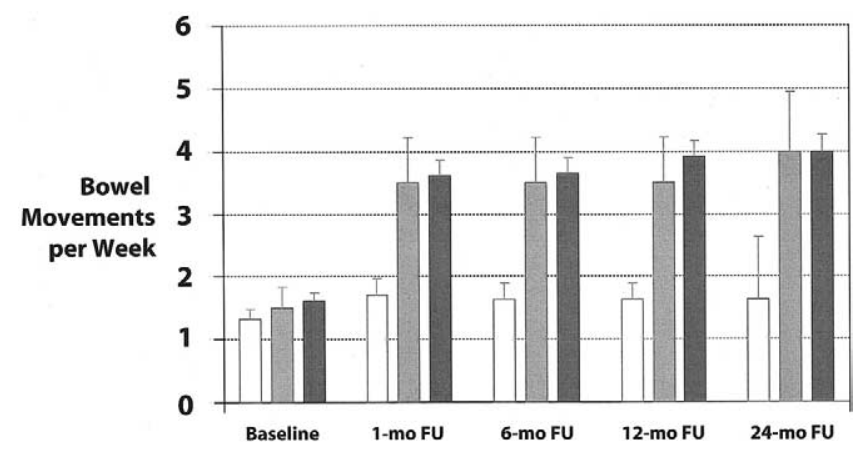

Figure 3. Bowel movements per week at baseline and each follow-up interval (mean $\pm \mathrm{SE}$ ). Open bars, STO group; gray bars, MIX group; black bars, PFD group. For each follow-up interval, STO versus PFD is significant at $P<.001$.

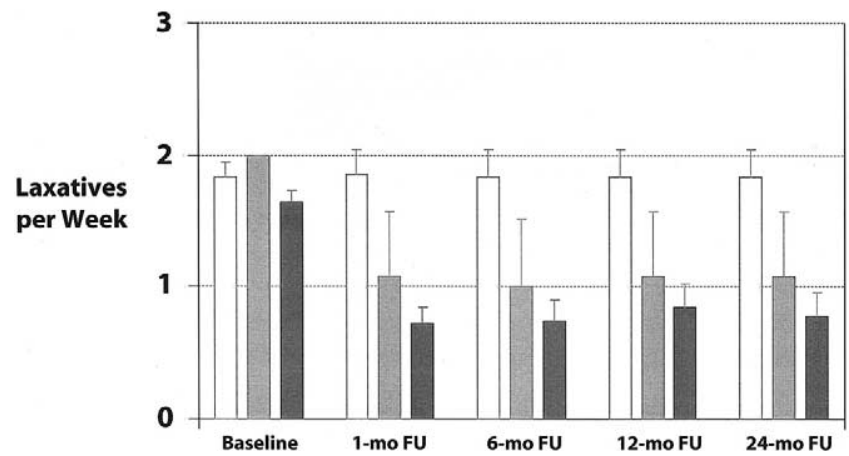

Figure 4. Average number of laxatives per week at baseline and each follow-up interval (mean \pm SE). Open bars, STO group; gray bars, MIX group; black bars, PFD group. For each follow-up interval, STO versus PFD is significant at $P<.001$.

\section{Laxative Use}

Figure 4 shows the average frequency of using laxatives at baseline and each follow-up interval. Repeated-measures ANCOVA showed that the interaction between groups and time period approached signficance (Greenhouse-Geisser F[4.51, 108.19] $=2.65 ; P=$ .032). Paired comparisons showed that the STO group was signficantly different from the PFD group at all follow-up intervals $(P<.01$ in each case), but the groups were not different at baseline. None of the patients in the trial were able to stop using laxatives altogether.

\section{Straining Frequency}

Figure 5 shows the average frequency of straining with bowel movements at baseline and each follow-up interval. Repeated-measures ANCOVA showed a significant interaction between groups and time period (Greenhouse-Geisser F[2.98, 71.59] $=4.09 ; P=.017$ ). Although Figure 5 suggests that patients in the PFD group strained less than patients in the STO group during follow-up, these differences were not statistically significant $(P>.05$ in each case). The significant inter-



Figure 5. Straining episodes per week at baseline and each follow-up interval (mean $\pm \mathrm{SE}$ ). The PFD group showed a greater reduction from baseline to follow-up than did the STO group. 


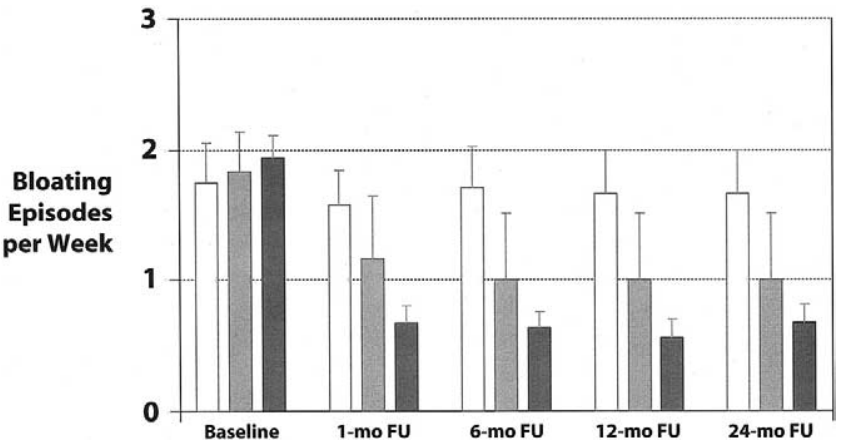

Figure 6. Bloating episodes per week at baseline and each follow-up interval (mean $\pm \mathrm{SE}$ ). Open bars, STO group; gray bars, MIX group; black bars, PFD group. For follow-up intervals 1, 6, and 12 months, STO versus PFD is significant at $P<.01$.

action is explained by the fact that the PFD group showed a greater reduction in straining from baseline than did the STO group.

\section{Bloating Frequency}

Figure 6 shows the average number of days per week with bloating at baseline and each follow-up interval. Repeated-measures ANCOVA showed a significant interaction between groups and time period (Greenhouse-Geisser $\mathrm{F}[3.05,73.30]=5.22 ; P=.002$ ). Paired comparisons showed that the groups were not different at baseline; however, at the 1-, 6-, 12-, and 24-month follow-up intervals, patients in the PFD group had significantly $(P \leq .015)$ less bloating than patients in the STO group.

\section{Pain Frequency}

Repeated-measures ANCOVA did not show a significant interaction between groups and time period (Geisser-Greenhouse corrected $\mathrm{F}[3.81,91.56]=0.29 ; P$ $=.875)$. Paired comparisons showed that patients in the PFD group reported less pain than patients in the STO group at all follow-up intervals $(P<.05$ in each case), but the groups were not different at baseline (data not shown).

\section{Whole Gut Transit Time}

Figure 7 shows the average number of Sitzmarks remaining in the colon on day 5 at baseline and at each follow-up interval. Repeated-measures ANCOVA showed a significant interaction between groups and time period (Greenhouse-Geisser F[2.32, 55.70] $=8.40 ; P<.001)$. Paired comparisons showed that patients in the PFD group had fewer Sitzmarks remaining on day 5 than patients in the STO group at every follow-up interval $(P<.001$ in each case). Patients in the PFD group also had fewer Sitzmarks remaining (ie, faster transit time) than patients in the STO group at baseline $(P=.015)$, but the differences between groups were significantly larger following biofeedback training.

When the Sitzmark test data were interpreted in terms of the proportion who had an abnormal transit test result (defined as $\geq 5$ Sitzmarks remaining in the bowel at day 5), there was no difference between the groups at baseline $(100 \%$ in all 3 groups by definition); however, at all follow-up intervals, transit was abnormally delayed in a significantly smaller proportion of patients in the PFD group compared with patients in the STO group: $41 \%$ versus $92 \%$ at 1 -month follow-up and $35 \%$ versus $92 \%$ at 6-, 12-, and 24-month follow-up.

Rectal stasis, defined as at least 10 of 20 Sitzmarks remaining in the bowel on day 5 with at least $80 \%$ of them in the sigmoid colon or rectum, was present in $35 \%$ (12 patients) with PFD, $17 \%$ (one patient) in the MIX group, and none of the STO group at baseline ( $P$ $=.046)$.

\section{Balloon Defecation Test}

Figure 8 shows the proportion of patients who were able to defecate a $50-\mathrm{mL}$ water-filled balloon within 5 minutes in each group at baseline and at each follow-up interval. By definition, none of the patients in the PFD group and all of the patients in the STO group could defecate the balloon at baseline. Following biofeedback training, $82 \%-85 \%$ of the PFD group could do so.

\section{Dyssynergia}

By definition, $100 \%$ of patients in the PFD group demonstrated dyssynergia at baseline and none of the patients in the STO group were dyssynergic. Following biofeedback training, the proportion of patients in the

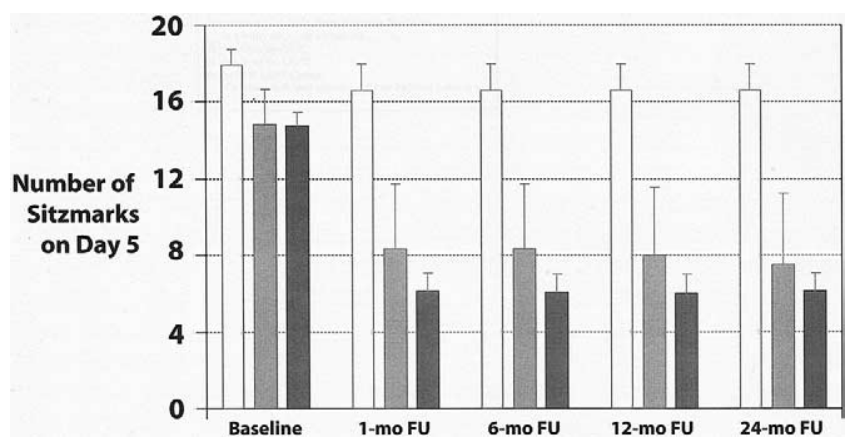

Figure 7. Number of Sitzmarks remaining on day 5 at baseline and each follow-up interval (mean \pm SE). Open bars, STO group; gray bars, MIX group; black bars, PFD group. For each follow-up interval, STO versus PFD is significant at $P<.001$. Five or more markers remaining defines an abnormally delayed transit. 


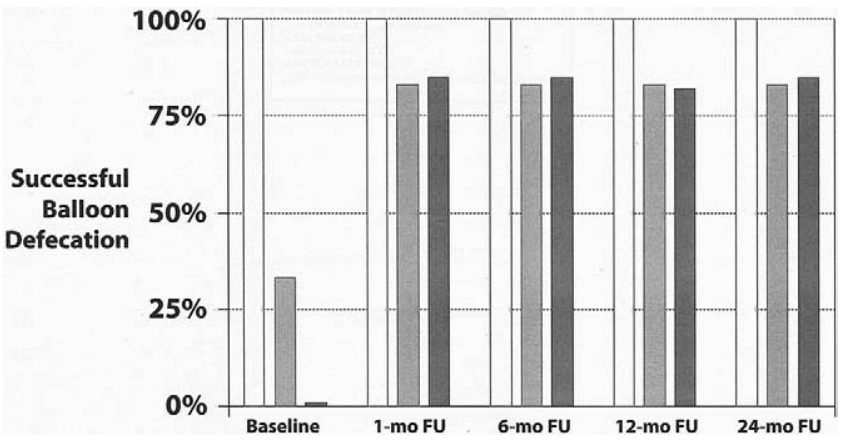

Figure 8. Percent of patients in each group who succeeded in defecating a 50-mL water-filled balloon within 5 minutes. Open bars, STO group; gray bars, MIX group; black bars, PFD group.

PFD group who were dyssynergic decreased from $100 \%$ to $9 \%$ at 6 months (Table 1). For the MIX group, 33\% were dyssynergic at baseline; this decreased to $17 \%$ by 6 months after biofeedback training.

\section{Mechanism of Biofeedback Training}

As predicted, satisfaction ratings were robustly correlated with reductions in dyssynergia at 1-month follow-up $(\rho=-0.69 ; P<.001)$ and 6 -month follow-up $(\rho=-0.70 ; P<.001)$ and with improvements in ability to defecate the water-filled balloon at 1-month follow-up $(\rho=.72 ; P<.001)$ and 6 -month follow-up $(\rho$ $=.73 ; P<.001)$. Satisfaction ratings were also correlated with increased straining pressure at 1 -month follow-up $(\rho=.36 ; P<.01)$ and 6 -month follow-up $(\rho=$ .36; $P=.01)$. The only other change in pelvic floor physiology that was correlated with satisfaction ratings was maximum tolerable volume; at 1-month follow-up, decreases in maximum tolerable volume correlated with satisfaction at a $\rho$ of $-0.28(P<.05)$. This correlation was not significant at 6 -month follow-up.
Table 1 shows physiologic data in each group at baseline and each follow-up interval. Baseline differences between the PFD group and the STO group were found for dyssynergia (present in all patients in the PFD group and none of the patients in the STO group by definition) and rectoanal inhibitory reflex threshold (lower in the PFD group than in the STO group). However, anorectal manometry identified no other differences between the STO group and the PFD group at baseline.

Following biofeedback training, patients in both the STO group and the PFD group showed significant decreases in urge threshold, but the reductions were greater in the PFD group. Following biofeedback training, the PFD group showed significant decreases in dyssynergia and significant increases in rectal pressure during straining, whereas the STO group showed no significant change in these parameters. The PFD group also showed significant reductions in maximum tolerable volume from baseline to follow-up, but patients in the STO group did not change their maximum tolerable volume.

\section{Physiologic Predictors of Successful Biofeedback Training}

Table 2 shows the correlations between satisfaction ratings at all follow-up intervals and all physiologic variables measured at baseline. Successful biofeedback training was predicted by all variables that reflect the presence of pelvic floor dyssynergia, including dyssynergia at baseline, inability to defecate a $50-\mathrm{mL}$ water-filled balloon, and rectal stasis. Satisfaction with biofeedback training was negatively correlated with the number of Sitzmarks remaining in the bowel on day 5 during baseline, suggesting that milder delays in whole gut transit were associated with better outcomes from biofeedback. No other anorectal parameter except rectal

Table 1. Physiologic Data at Baseline and Follow-up Intervals

\begin{tabular}{|c|c|c|c|c|c|c|c|c|c|c|c|c|c|}
\hline & \multicolumn{2}{|c|}{$\begin{array}{l}\text { Anal canal } \\
\text { resting pressure } \\
(m L)\end{array}$} & \multicolumn{2}{|c|}{$\begin{array}{c}\text { Rectoanal } \\
\text { inhibitory reflex } \\
\text { threshold }(m L)\end{array}$} & \multicolumn{2}{|c|}{$\begin{array}{l}\text { Urge threshold } \\
\qquad(m L)\end{array}$} & \multicolumn{2}{|c|}{$\begin{array}{c}\text { Maximum tolerable } \\
\text { pressure }(\mathrm{mm} \mathrm{Hg})\end{array}$} & \multicolumn{2}{|c|}{$\begin{array}{c}\text { Compliance } \\
\text { (mm Hg@100 } \\
\text { mL) }\end{array}$} & \multicolumn{2}{|c|}{$\begin{array}{c}\text { Straining rectal } \\
\text { pressure }(\mathrm{mm} \\
\mathrm{Hg})\end{array}$} & \multirow{2}{*}{$\begin{array}{c}\text { Mean } \\
\text { dyssynergia } \\
(\%)\end{array}$} \\
\hline & Mean & SD & Mean & SD & Mean & SD & Mean & SD & Mean & SD & Mean & SD & \\
\hline \multicolumn{14}{|l|}{ STO group } \\
\hline Baseline & 60.67 & 12.05 & 17.50 & 4.52 & 108.33 & 35.89 & 279.17 & 65.57 & 11.92 & 3.03 & 45.92 & 12.92 & 0 \\
\hline 1-month follow-up & 61.33 & 13.51 & 17.50 & 4.52 & $75.00^{a}$ & 26.11 & 258.22 & 55.73 & 12.17 & 2.92 & 50.67 & 6.41 & 0 \\
\hline 6-month follow-up & 61.82 & 12.87 & 16.36 & 5.05 & $72.73^{a}$ & 26.11 & 254.55 & 56.81 & 12.45 & 3.05 & 51.91 & 6.46 & 0 \\
\hline \multicolumn{14}{|l|}{ MIX group } \\
\hline Baseline & 70.33 & 14.73 & 13.33 & 5.16 & 108.33 & 20.41 & 283.33 & 40.83 & 12.67 & 3.08 & 33.00 & 21.33 & 33 \\
\hline 1-month follow-up & 70.17 & 14.58 & 15.00 & 5.48 & 58.33 & 20.41 & $241.67^{a}$ & 37.64 & 12.33 & 2.81 & 51.17 & 13.45 & 17 \\
\hline 6-month follow-up & 73.60 & 10.24 & 14.00 & 5.48 & 50.00 & 0 & $230.00^{a, b}$ & 27.39 & 12.00 & 2.91 & 52.00 & 17.07 & 17 \\
\hline \multicolumn{14}{|l|}{ PFD group } \\
\hline Baseline & 66.06 & 12.28 & $13.82^{b}$ & 5.51 & 100.00 & 34.82 & 270.59 & 66.42 & 13.06 & 2.99 & 36.71 & 14.64 & $100^{b}$ \\
\hline 1-month follow-up & 66.21 & 11.54 & $13.82^{b}$ & 4.93 & $51.47^{a, b}$ & 8.57 & $219.12^{\mathrm{a}, \mathrm{b}}$ & 38.95 & 13.53 & 2.80 & $49.53^{a}$ & 10.27 & $9^{a}$ \\
\hline 6-month follow-up & 66.41 & 12.10 & 13.53 & 4.85 & $51.47^{a, b}$ & 8.57 & $211.76^{a, b}$ & 30.30 & 13.62 & 2.81 & $50.97^{a}$ & 10.89 & $9^{a}$ \\
\hline
\end{tabular}

${ }^{a} P<.05$ compared with baseline for same group (within subjects).

${ }^{b} P<.01$ compared with STO group for same time point (between groups). 
Table 2. Baseline Predictors of Satisfaction With Biofeedback Training

\begin{tabular}{|c|c|c|c|c|}
\hline & \multicolumn{4}{|c|}{ Follow-up } \\
\hline & 1 Month & 6 Months & 12 Months & 24 Months \\
\hline \multicolumn{5}{|l|}{ Physiologic variables } \\
\hline Stasis & $0.34^{a}$ & $0.36^{b}$ & $0.39^{b}$ & $0.39^{b}$ \\
\hline Balloon defecation & $-0.58^{b}$ & $-0.58^{b}$ & $-0.49^{b}$ & $-0.49^{b}$ \\
\hline Dyssynergia & $0.56^{b}$ & $0.56^{b}$ & $0.51^{a}$ & $0.47^{b}$ \\
\hline No. of Sitzmarks & $-0.56^{b}$ & $-0.55^{b}$ & $-0.58^{b}$ & $-0.56^{b}$ \\
\hline Resting anal canal pressure & 0.25 & 0.20 & 0.23 & 0.16 \\
\hline Rectoanal inhibitory reflex threshold & -0.12 & -0.10 & -0.09 & -0.12 \\
\hline Urge threshold & -0.15 & -0.17 & -0.18 & -0.20 \\
\hline Maximum tolerable volume & -0.03 & -0.10 & -0.11 & -0.07 \\
\hline Compliance & $0.29^{a}$ & $0.33^{a}$ & $0.32^{a}$ & $0.31^{a}$ \\
\hline Straining rectal pressure & -0.09 & -0.14 & -0.12 & -0.16 \\
\hline \multicolumn{5}{|l|}{ Clinical variables (/wk) } \\
\hline Bowel movements & $0.35^{a}$ & $0.33^{a}$ & $0.39^{b}$ & $0.36^{b}$ \\
\hline Laxatives & $-0.37^{b}$ & $-0.35^{a}$ & $-0.34^{a}$ & $-0.38^{b}$ \\
\hline Pain & $-0.33^{a}$ & $-0.32^{a}$ & $-0.28^{a}$ & $-0.32^{a}$ \\
\hline Bloating & -0.13 & -0.06 & -0.02 & 0.07 \\
\hline Straining & 0.16 & 0.16 & 0.11 & 0.12 \\
\hline
\end{tabular}

NOTE. The correlations between satisfaction ratings and clinical variables measured at baseline are shown. Significant correlations were found, which showed that more frequent bowel movements during baseline and taking laxatives less often were predictive of greater success from biofeedback training. More frequent pain episodes during baseline was associated with a poorer response to biofeedback training. An ordinal regression analysis, which adjusts for the intercorrelations among the predictor variables, showed that the Nagelkerke $\mathrm{R}^{2}$ value was $0.314(P$ $<.001)$, and the significant independent predictors were number of weekly laxative doses taken $(P=.004)$ and number of weekly pain episodes $(P=.013)$.

ap $<.05$.

${ }^{b} P<.01$

compliance was associated with the success of biofeedback training; greater compliance was associated (at the $P<.05$ level) with better outcomes. To adjust for intercorrelations among the physiologic predictor variables in Table 2, an ordinal regression analysis was performed that included all the baseline variables in Table 2 for which the univariate correlations were statistically significant. The Nagelkerke $\mathrm{R}^{2}$ value was 0.646 $(P<.001)$, and the significant independent predictors were number of Sitzmarks remaining in the colon after 5 days $(P<.001)$, stasis in the rectum $(P=.011)$, and balloon defecation $(P=.024)$.

\section{Clinical Predictors of Successful Biofeedback Training}

Table 2 shows the correlations between satisfaction ratings and clinical variables measured at baseline. Significant correlations were found, which showed that more frequent bowel movements during baseline and taking laxatives less often were predictive of greater success from biofeedback training. More frequent pain episodes during baseline were associated with a poorer response to biofeedback training. An ordinal regression analysis, which adjusts for the intercorrelations among the predictor variables, showed that the Nagelkerke $\mathrm{R}^{2}$ value was $0.314(P<.001)$, and the significant independent predictors were number of weekly laxative doses taken $(P=.004)$ and number of weekly pain episodes $(P$ $=.013)$.

\section{Discussion}

\section{Effectiveness of Biofeedback Training in Slow Transit Constipation}

This study shows that, in contrast to recently reported views, biofeedback is more effective for the treatment of outlet dysfunction-type constipation than it is for the treatment of slow transit constipation. Seventyone percent of patients with PFD reported "fair" or "major" satisfaction with the degree of improvement in their symptoms of constipation following biofeedback training compared with only $8 \%$ (one patient) in the STO group and $50 \%$ of the MIX group. These subjective reports of improvement were supported by symptom diary data showing that patients in the PFD group experienced greater improvements than patients in the STO group in stool frequency (Figure 3), laxative use (Figure 4), straining (Figure 5), and bloating (Figure 6). Patients in the PFD group were also found to show greater improvements in transit time (Figure 7) and the occurrence of dyssynergic patterns on straining to defecate.

The MIX group included 6 patients who could not be definitively classified as having pelvic floor dyssynergia 
because they met only one of the 2 criteria for this group. However, as shown in Figures 2-8, they were more similar to the PFD group than to the STO group; they showed improvements in satisfaction ratings, stool frequency, laxative use, straining, bloating, transit time, and ability to defecate a water-filled balloon that were comparable to patients in the PFD group. Based on these similarities, it is likely that patients who show either inability to defecate a $50-\mathrm{mL}$ water-filled balloon or paradoxical contraction of the pelvic floor on manometry could benefit from biofeedback training and should be offered this treatment.

These data also provide an explanation for the differences between our conclusions and those of the St Mark's group $^{18,19}$ and Wang et al. ${ }^{20}$ These other investigators defined slow transit constipation exclusively by the Sitzmark whole gut transit study and diagnosed outlet dysfunction in any patient who demonstrated paradoxical contraction of the pelvic floor or failure to relax during straining to defecate; these 2 diagnostic classifications (ie, whether the patient had slow transit and whether the patient had pelvic floor dyssynergia) were made independently of each other. In designing our study, we initially made the same assumption, that is, that these 2 diagnostic classifications could be made independently of each other. We therefore enrolled only patients who had abnormal whole gut transit studies ( $\geq 5$ of 20 Sitzmarks remaining at day 5), and we further classified the same patients as having or not having pelvic floor dyssynergia based on both (1) an anorectal manometry study result demonstrating dyssynergia and (2) a balloon defecation test result demonstrating inability to defecate a $50-\mathrm{mL}$ water-filled balloon. However, our data suggest that this assumption was incorrect and that patients with delayed whole gut transit plus pelvic floor dyssynergia did not have true slow transit constipation despite an abnormal Sitzmark study result. This is shown by the fact that $65 \%$ of patients with pelvic floor dyssynergia plus abnormally delayed Sitzmark studies normalized their transit times when they were treated with biofeedback, whereas only $22 \%$ of patients with isolated slow transit normalized their Sitzmark studies following biofeedback training. This suggests that pelvic floor dyssynergia was the cause of the delayed transit and that the Sitzmark study cannot be interpreted as a diagnostic marker for slow transit constipation in patients who have pelvic floor dyssynergia.

The mechanism by which pelvic floor dyssynergia causes delayed whole gut transit was not definitively identified in our study. One possibility is that distention of the rectum with stool causes a reflex inhibition of colonic motility, as has been suggested by Mollen et al. ${ }^{24}$
There is evidence that distention of the rectum causes a reflex inhibition of gastric emptying, ${ }^{25}$ so reflex inhibition of colon motility is plausible. An alternative explanation is a simple stopper effect; if the only outlet available to the colon is obstructed, fecal material will have to back up. This last possibility is consistent with the observation that $35 \%$ of patients in the PFD group demonstrated rectal stasis (at least 10 of 20 Sitzmarks retained and at least $80 \%$ of these in the sigmoid colon or rectum) compared with none of the patients with rectal stasis in the STO group. However, the fact that $65 \%$ of patients in the PFD group did not meet criteria for rectal stasis lends weight to the hypothesis that reflex inhibition of pancolonic motility may be responsible for delayed transit in patients with PFD. To answer this question more definitively, studies are needed in which proximal colon motility is measured during prolonged obstruction of defecation or prolonged distention of the rectum. Such studies could provide new insights into the pathophysiologic mechanisms for subtypes of constipation.

\section{Maintenance of Biofeedback Training Effects}

There has also been controversy about the longterm benefits of biofeedback training for the treatment of outlet dysfunction-type constipation. Some have reported good maintenance of biofeedback effects at follow-up 1 year $^{26}$ or 2 years ${ }^{18}$ following biofeedback taining, whereas others have described nearly a complete regression to baseline levels of symptoms by 1 year. ${ }^{27}$ Battaglia et $\mathrm{al}^{28}$ divided their patients into slow transit and outlet dysfunction types of constipation and reported good maintenance at 2 years for the outlet dysfunction group but not for the slow transit group. Our data support the findings of Battaglia et al; we observed excellent retention of treatment gains in the PFD group (Figures 2-6), with $71 \%$ reporting "fair" or "major" improvement and $65 \%$ having normal whole gut transit times at 24-month follow-up.

\section{Mechanism of Biofeedback Training}

Biofeedback training is designed to teach patients to relax the pelvic floor (rather than paradoxically contracting it) while straining to defecate. The changes in physiologic measures from baseline to 1-month follow-up and 6-month follow-up suggest that these objectives were achieved. When all subjects were pooled and correlations were computed between satisfaction with treatment and changes in physiologic parameters, greater satisfaction was associated with elimination of dyssynergia ( $\rho=-0.69$ at 1 month; $\rho=-0.70$ at 6 months), 
improved ability to defecate a $50-\mathrm{mL}$ water-filled balloon ( $\rho=0.72$ at 1 month; $\rho=0.73$ at 6 months), and increases in the pressure measured in the rectum when patients strained to defecate ( $\rho=0.36$ at 1 month; $\rho=$ 0.36 at 6 months). We also observed that greater satisfaction with biofeedback treatment was associated with improvements in transit time, but this may be an artifact. As already noted, $65 \%$ of patients in the PFD group but only $8 \%$ of patients in the STO group showed normalization of transit time following successful treatment of pelvic floor dyssynergia, suggesting that delayed transit in the PFD group was secondary to pelvic floor dyssynergia rather than being due to a primary colon motility disorder.

Table 1 shows changes in anorectal parameters from baseline to follow-up for the PFD group and the STO group separately. Consistent with the correlations in the pooled sample, both the PFD group and the STO group showed reductions in dyssynergia and increases in rectal pressure during straining, which were the intended effects of biofeedback. Both groups also showed decreases in the threshold volume of rectal distention required to elicit an urge to defecate, and the PFD group showed a significant reduction in maximum tolerable volume. These changes in sensory thresholds were unanticipated and are believed to reflect the effects of eliminating or reducing the chronic distention of the rectum with retained stool.

\section{Predictors of Outcome From Biofeedback Training}

Importantly, a group of physiologic variables that identify patients who have pelvic floor dyssynergia predicted the response to biofeedback training. These variables were dyssynergia on manometry, inability to defecate a water-filled balloon, and rectal stasis (retention of stool in the rectum). This supports the hypothesis that biofeedback training to teach relaxation of the pelvic floor only benefits those patients who have an objectively measurable dysfunction of the pelvic floor. A second group of variables that were correlated with the outcome of biofeedback training appear to identify patients who had more severe constipation; they did more poorly. These variables included a greater number of retained Sitzmarks during the transit study and fewer bowel movements and more frequent use of laxatives during baseline. Interestingly, frequency of reporting straining to defecate did not predict response to pelvic floor biofeedback.

Frequency of experiencing abdominal pain predicted a poor response to biofeedback. This was an unexpected finding and is unexplained. It may reflect patients who have irritable bowel syndrome in addition to chronic constipation.

\section{Study Limitations}

The effectiveness of pelvic floor biofeedback training depends in part on the skills of the biofeedback therapist and the particular techniques used to perform the training. The biofeedback training procedures used in this study are representative of what has been described in the literature, but they differ from the techniques used by some laboratories. Moreover, all patients were treated by one physician who is highly experienced in biofeedback training. It is unknown whether similarly good outcomes will be obtained in other research or clinical settings. Nevertheless, these study findings show that substantial clinical benefits can be achieved in suitably selected patients and that they can be maintained for at least 2 years.

\section{Specificity of Biofeedback Training Effects}

Norton et $\mathrm{al}^{29}$ recently reported the results of a study comparing biofeedback with standard care and advice for the treatment of fecal incontinence. They found no differences in patients' ratings of their satisfaction with treatment and no differences in the frequency of incontinence following treatment. Anal canal squeeze pressure and anal canal resting pressure improved in all patients, but improvement was not related to whether the patients received biofeedback training. These investigators concluded that biofeedback provides no greater benefit than standard care supplemented by advice and education. Our data suggest that, for outlet dysfunction constipation at least, biofeedback does provide a specific benefit. First, $71 \%$ of patients with outlet dysfunctiontype constipation reported that they were somewhat or greatly improved, and improvement ratings were substantiated by increases in the frequency of bowel movements and decreases in laxative use and straining. Second, improvement was related to the etiology of the complaint of constipation; only patients with pelvic floor dyssynergia and inability to defecate a water-filled balloon benefited, and benefits were strongly correlated with improvement in the ability to relax the pelvic floor during defecation and improvement in the ability to defecate a water-filled balloon. Third, improvements were well sustained for the 2 years that these patients were followed up after treatment.

\section{References}

1. Higgins PD, Johanson JF. Epidemiology of constipation in North America: a systematic review. Am J Gastroenterol 2004;99:750759. 
2. Whitehead WE, Wald A, Diamant NE, Enck P, Pemberton JH, Rao SSC. Functional disorders of the anus and rectum. In: Drossman DA, Corazziari E, Talley NJ, Thompson WG, Whitehead WE, eds. Rome II: the functional gastrointestinal disorders. 2nd ed. McLean, VA: Degnon Associates, 2000:483-532.

3. Bassotti G, Chistolini F, Marinozzi G, Morelli A. Abnormal colonic propagated activity in patients with slow transit constipation and constipation-predominant irritable bowel syndrome. Digestion 2003;68:178-183.

4. Bassotti G, Chistolini F, Nzepa FS, Morelli A. Colonic propulsive impairment in intractable slow-transit constipation. Arch Surg 2003;138:1302-1304.

5. He CL, Burgart L, Wang L, Pemberton J, Young-Fadok T, Szurszewski J, Farrugia G. Decreased interstitial cell of cajal volume in patients with slow-transit constipation. Gastroenterology 2000; 118:14-21.

6. Wedel T, Spiegler J, Soellner S, Roblick UJ, Schiedeck TH, Bruch $\mathrm{HP}$, Krammer HJ. Enteric nerves and interstitial cells of Cajal are altered in patients with slow-transit constipation and megacolon. Gastroenterology 2002;123:1459-1467.

7. Locke GR III, Pemberton JH, Phillips SF. American Gastroenterological Association Medical Position Statement: guidelines on constipation. Gastroenterology 2000;119:1761-1766.

8. Locke GR III, Pemberton JH, Phillips SF. AGA technical review on constipation. American Gastroenterological Association. Gastroenterology 2000;119:1766-1778.

9. Preston DM, Lennard-Jones JE. Anismus in chronic constipation. Dig Dis Sci 1985;30:413-418.

10. Kuijpers HC, Bleijenberg G. The spastic pelvic floor syndrome. A cause of constipation. Dis Colon Rectum 1985;28:669-672.

11. Bleijenberg G, Kuijpers HC. Treatment of the spastic pelvic floor syndrome with biofeedback. Dis Colon Rectum 1987;30:108111.

12. Heymen S, Jones KR, Scarlett Y, Whitehead WE. Biofeedback treatment of constipation: a critical review. Dis Colon Rectum 2003;46:1208-1217.

13. Duthie GS, Bartolo DC. Anismus: the cause of constipation? Results of investigation and treatment. World J Surg 1992;16: 831-835.

14. Rao SS, Welcher KD, Leistikow JS. Obstructive defecation: a failure of rectoanal coordination. Am J Gastroenterol 1998;93: 1042-1050.

15. Rao SS. Biofeedback therapy for dyssynergic (obstructive) defecation. J Clin Gastroenterol 2000;30:115-116.

16. Enck P. Biofeedback training in disordered defecation. A critical review. Dig Dis Sci 1993;38:1953-1960.

17. Koutsomanis D, Lennard-Jones JE, Roy AJ, Kamm MA. Controlled randomised trial of visual biofeedback versus muscle training without a visual display for intractable constipation. Gut 1995; 37:95-99.
18. Chiotakakou-Faliakou E, Kamm MA, Roy AJ, Storrie JB, Turner IC. Biofeedback provides long-term benefit for patients with intractable, slow and normal transit constipation. Gut 1998;42:517521.

19. Emmanuel AV, Kamm MA. Response to a behavioural treatment, biofeedback, in constipated patients is associated with improved gut transit and autonomic innervation. Gut 2001;49:214-219.

20. Wang J, Luo MH, Qi QH, Dong ZL. Prospective study of biofeedback retraining in patients with chronic idiopathic functional constipation. World J Gastroenterol 2003;9:2109-2113.

21. Hinton JM, Lennard-Jones JE, Young AC. A new method for studying gut transit times using radioopaque markers. Gut 1969;10: 842-847.

22. Rao SS, Welcher KD, Leistikow JS. Obstructive defecation: a failure of rectoanal coordination. Am J Gastroenterol 1998;93: 1042-1050.

23. SPSS advanced statistics [computer program]. Version 11.0. Chicago, IL: SPSS Inc, 2003.

24. Mollen RM, Salvioli B, Camilleri M, Burton D, Kost LJ, Phillips SF, Pemberton $\mathrm{JH}$. The effects of biofeedback on rectal sensation and distal colonic motility in patients with disorders of rectal evacuation: evidence of an inhibitory rectocolonic reflex in humans? Am J Gastroenterol 1999;94:751-756.

25. Youle MS, Read NW. Effect of painless rectal distension on gastrointestinal transit of solid meal. Dig Dis Sci 1984;29:902906.

26. Wiesel PH, Dorta G, Cuypers P, Herranz M, Kreis ME, Schnegg JF, Jornod P. Patient satisfaction after biofeedback for constipation and pelvic floor dyssynergia. Swiss Med Wkly 2001;131:152166.

27. Ferrara A, De Jesus S, Gallagher JT, Williamson PR, Larach SW, Pappas D, Mills J, Sepulveda JA. Time-related decay of the benefits of biofeedback therapy. Tech Coloproctol 2001;5:131-135.

28. Battaglia E, Serra AM, Buonafede G, Dughera L, Chistolini F, Morelli A, Emanuelli G, Bassotti G. Long-term study on the effects of visual biofeedback and muscle training as a therapeutic modality in pelvic floor dyssynergia and slow-transit constipation. Dis Colon Rectum 2004;47:90-95.

29. Norton C, Chelvanayagam S, Wilson-Barnett J, Redfern S, Kamm MA. Randomized controlled trial of biofeedback for fecal incontinence. Gastroenterology 2003;125:1320-1329.

Received August 19, 2004. Accepted April 8, 2005.

Address requests for reprints to: Giuseppe Chiarioni, MD, Divisione di Riabilitazione Gastroenterologica, Universitá di Verona, Azienda Ospedaliera di Verona, Centro Ospedaliero Clinicizzato, 37067 Valeggio sul Mincio (Verona), Italy. e-mail: chiarioni@tin.it; fax: (39) 0457950188.

Supported in part by National Institute of Diabetes and Digestive and Kidney Diseases grants R01 DK57048 and R24 DK67674. 